\title{
ANALYSIS OF CHARGE VERSUS THE SURFACE FERMI LEVEL POSITION AT SILICON SURFACE
}

\author{
B. Ad八MOWICZ \\ Institute of Physics, Silesian Technical University \\ Krrzywoustego 2, 44-100 Gliwice, Poland \\ (Received May 21, 1991; in revised form July 15, 1991)
}

The surface charge $Q_{\text {sc }}$ versus the surface Fermi level position $\Phi_{\mathrm{s}}$ for real (111) surfaces of $p$ - and $n$-type Si has been studied. The values of $Q_{\text {sc }}$ have been obtained on the basis of measurements of the surface potential $V_{s}$ by means of the surface photovoltage method. The complementary character of $Q_{\mathrm{sc}}\left(\Phi_{\mathrm{s}}\right)$ dependences for the surfaces of $p$ - and $n$-type Si supports the fact that the surface state distribution is not dependent on the type of bulk doping but on the surface preparation process.

PACS numbers: $73.20 .-\mathrm{r}$

\section{Introduction}

The charge accumulated near the semiconductor surface plays an important part in the action of microelectronic devices [1]. On the other hand its changes versus the surface Fermi level position deliver information about the occupation and distribution of the electron surface states. In this work the surface space charge $Q_{\mathrm{sc}}$, appearing on the real silicon surface after chemical etching as well as vacuum baking at 700,920 and $970 \mathrm{~K}$, has been examined. The values of $Q_{\text {sc }}$ at different temperatures have been calculated on the basis of the surface potential $V_{\mathbf{s}}$, estimated from the saturation of the surface photovoltage $\Delta V_{\mathbf{s}}$ for the large intensity of light. The graphical analysis of the $Q_{\mathrm{sc}}\left(\Phi_{\mathbf{s}}\right)$ dependences has proved that the system of surface states both for $n$ - and $p$-type $S i$ is very similar and mainly depends on the surface preparation process.

\section{Experimental}

The examined samples were of $p$-type $(\rho=15 \Omega \mathrm{m})$ and $n$-type $(\rho=11 \Omega \mathrm{m})$ silicon slices with dimensions of $12 \times 5 \times 0.28 \mathrm{~mm}$. Before doing measurements the samples were cleaned in organic reagents and etched in a $46 \%$ IIF solution with subsequent rinsing in acetone and deionized water. Then a part of the samples was baked in the vacuum of $10^{-4} \mathrm{~Pa}$ in order to change the surface condition. Next the 
samples were mounted in the measuring chamber under the same pressure. Before recording the surface photovoltage the samples were baked additionally at $470 \mathrm{~K}$ for $1 \mathrm{~h}$ and cooled to room temperature.

The surface photovoltage was detected in the static capacitor system using modulated light with a frequency of $40 \mathrm{~Hz}$. The details of the measuring set-up are described in Refs. [2,3]. The reference electrode was a semitransparent gold layer evaporated on the glass plate. The distance between the examined surface and the electrode was about $40 \mu \mathrm{m}$. The noise level of $\Delta V_{\mathrm{s}}$ amounted to $50 \mu V$. The surface photovoltage was generated by a $50 \mathrm{~W}$ tungsten lamp with focused beam.

Additionally the measurements of the dark conductivity, photoconductivity and Hall effect were carried out to determine the bulk concentrations and mobilities of carriers as well as the value of the Dember photovoltage.

The temperature of the sample was changed from $300 \mathrm{~K}$ to $430 \mathrm{~K}$ (at a rate of $1 \mathrm{~K} / \mathrm{min}$ ) by heating the base alumina plate.

\section{Results and discussion}

The measurements of the surface photovoltage $\Delta V_{\mathrm{s}}$ versus the light intensity allowed determination of the surface potential $V_{\mathrm{s}}$, from the saturation of $\Delta V_{\mathbf{s}}$ for the large intensities, after subtraction of the Dember photovoltage $U_{\mathrm{D}}$. The value of $U_{\mathrm{D}}$ was calculated using formula (3.1) [1] and the measured values of the dark conductivity and photoconductivity of the examined samples:

$$
U_{\mathrm{D}}=\frac{k T}{q} \frac{\mu_{n}-\mu_{p}}{\mu_{n}+\mu_{p}} \ln \frac{\sigma^{*}}{\sigma}
$$

where $q$ was electronic charge, $k$ - Boltzmann constant, $T$ - temperature, and $\mu_{n}, \mu_{p}$ were the electron and hole mobilities, and $\sigma^{*}, \sigma-$ conductivities of the sample in the dark and under illumination, respectively.

The results of $U_{\mathrm{D}}$ calculations for $p$-type samples are presented in Fig. 1. Similar dependences were obtained for $n$-type silicon.

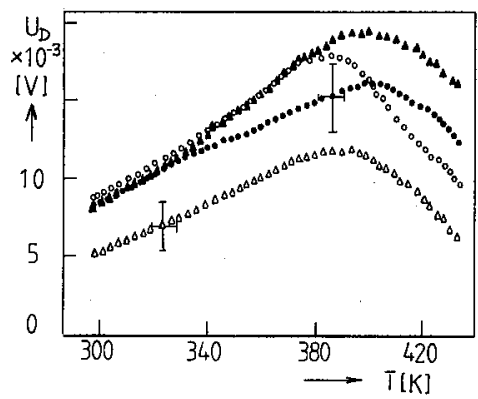

Fig. 1. Experimental dependences of the Dember photovoltage versus temperature for p-type Si: etched surface (o), baked at $700 \mathrm{~K}(\bullet), 920 \mathrm{~K}(\triangle)$ ), and $970 \mathrm{~K}$ (full triangle).

In Fig. 2 the temperature dependences of the surface potential $V_{\mathrm{s}}$ for $p$-and $n$-type Si after different surface treatments are shown. The values of $V_{s}$ positive 
for $p$-type and negative for $n$-type Si prove that at $300 \mathrm{~K}$ the used preparation technology (chemical etching and vacuum baking) generates the depletion layer near the surface, regardless of the type of bulk doping. The positive values of $V_{\mathbf{s}}$
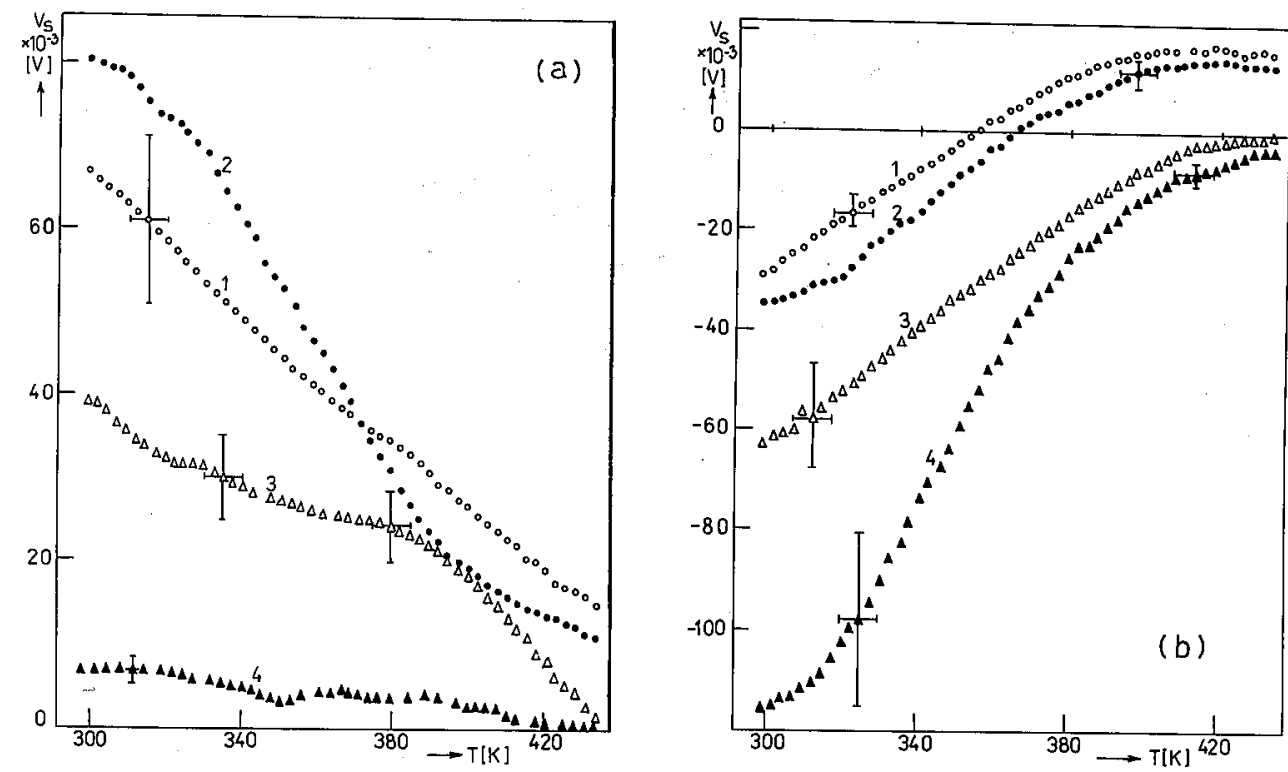

Fig. 2. Experimental dependences of the surface potential on temperature for $p$ - (a) and $n$-type Si (b); etched surface (1), baked at $700 \mathrm{~K}(2), 920 \mathrm{~K}(3)$ and $970 \mathrm{~K}$ (4).

for the $p$-type samples (Fig. 2a) lead to an argument for an existence of positive charge in surface states of donor character. Whereas the negative values of $V_{\mathbf{s}}$ for $n$-type Si (Fig. 2b) prove that the acceptor surface are present on this surface. A change in the sign of $V_{\mathrm{s}}$ at certain temperatures for the surfaces etched and baked at $700 \mathrm{~K}$ (curve 1 and 2 in Fig. 2b) shows the possibility of existence of the donor surface states simultaneously with the acceptor ones. An increase of temperature causes a change in occupation of surface states and an increasing contribution of donor states to the total surface charge.

Then the values of $V_{\mathrm{s}}$ were used for calculations of the Fermi level position $\Phi_{\mathrm{s}}$ from the relation: $\Phi_{\mathrm{s}}=\Phi_{\mathrm{b}}+V_{\mathrm{s}}$, where $\Phi_{\mathrm{b}}=\left(E_{\mathrm{F}}-E_{\mathrm{i}}\right) / q$ and $E_{\mathrm{F}}, E_{\mathrm{i}}$ were Fermi energies in bulk of doped or intrinsic semiconductor respectively. $\Phi_{\mathrm{b}}$ as a function of temperature was computed using the usual relations and estimated values of carrier concentrations from the measurements of the dark conductivity and Hall effect at different temperatures.

The dependences of $\Phi_{\mathrm{s}}$ and $\Phi_{\mathrm{b}}$ on temperature are shown in Fig. 3. We see that the Fermi level on the surface is generally not pinned with temperature, contrary to the case of the clean Si surface [4], but on the other hand the changes on $\Phi_{\mathrm{s}}$ are slower than $\Phi_{\mathrm{b}}$. The points of stabilization of $\Phi_{\mathrm{s}}$ at higher temperatures, close to the midgap, support the literature conclusion that the Fermi level, if pinned 


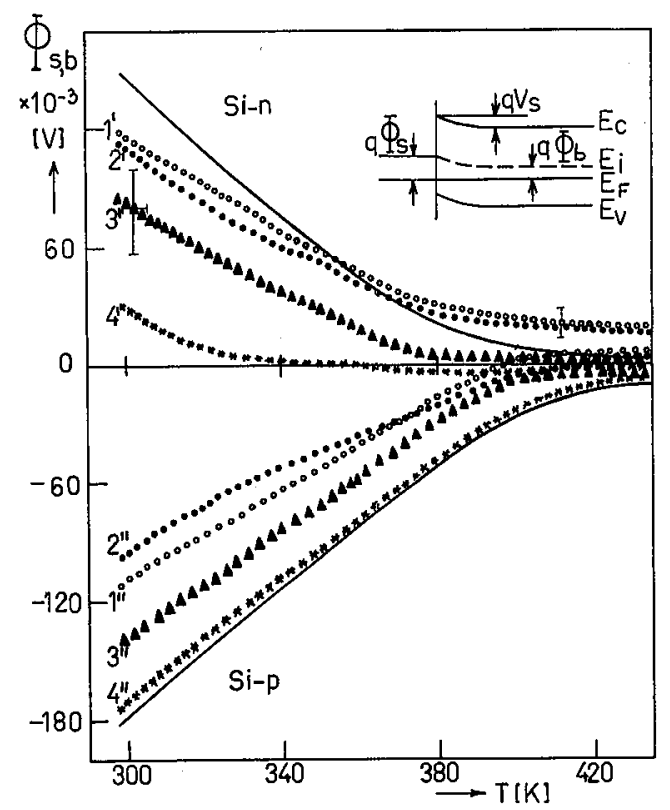

Fig. 3. Surface position $\Phi_{\mathrm{s}}$ (points) and bulk position $\Phi_{\mathrm{b}}$ (solid lines) of the Fermi level for $p$ - $\left({ }^{\prime \prime}\right)$ and $n$-type $\left({ }^{\prime}\right) \mathrm{Si}$; etched surface (1), baked at $700 \mathrm{~K}(2), 920 \mathrm{~K}(3)$ and $970 \mathrm{~K}$ (4).

on the real surface, is located usually around the midgap [5]. Such behaviour of $E_{\mathrm{F}}$ is associated with the surface states, originating from the dangling bonds and defects as well as impurities and lattice imperfections, changing their occupation with the changes of the temperature. From the energetical point of view the surface states on the real semiconductor surface create two branches in the band gap of donor and acceptor types with a density expressed by the following relations:

$$
N_{\mathrm{sa}}=A \exp (a E / k T) \quad \text { and } \quad N_{\mathrm{sd}}=B \exp (-b E / k T)
$$

where $A, B, a, b$ are the coefficients of the distribution function, and $E$ is the energy (zero in the midgap) [6].

Some information about the charge $Q_{s s}$ and the distribution of the surface states may be obtained by means of an analysis of the surface space charge $Q_{\mathbf{s c}}$ characteristics due to the fact that the neutrality condition should be maintained near the surface $\left(\Delta Q_{\mathrm{sc}}=-\Delta Q_{\mathrm{ss}}\right)$. The values of $Q_{\mathrm{sc}}$ were calculated from the following formula [1]:

where

$$
Q_{\mathrm{sc}}= \pm q\left(n_{\mathrm{b}}+p_{\mathrm{b}}\right) L_{\mathrm{D}} F_{\mathrm{s}}
$$

$$
L_{\mathrm{D}}=\left\{\varepsilon \varepsilon_{0} k T /\left[q^{2}\left(n_{\mathrm{b}}+p_{\mathrm{b}}\right)\right]\right\}^{1 / 2},
$$

$\varepsilon, \varepsilon_{0}$ were semiconductor and free space permittivities, $n_{\mathrm{b}}$ and $p_{\mathrm{b}}$ were bulk concentrations of electrons and holes respectively,

$$
F_{\mathrm{s}}=\sqrt{2}\left[\cosh \left(u_{\mathrm{b}}+v_{\mathrm{s}}\right) / \cosh u_{\mathrm{b}}-v_{\mathrm{s}} \tanh u_{\mathrm{b}}-1\right]^{1 / 2},
$$




$$
u_{\mathrm{b}}=q \Phi_{\mathrm{b}} / k T, \quad \text { and } \quad v_{\mathrm{s}}=q V_{\mathrm{s}} / k T
$$

The results of calculations of $Q_{\mathrm{sc}}$ versus $\Phi_{\mathrm{s}}$ are shown in Fig. 4. The clearly complementary character of these curves, for $p$ - and $n$-type $\mathrm{Si}$ after the same technological treatment is visible. It suggests that the distribution function of the surface states does not depend on the bulk doping but mainly on the surface preparation process. Moreover we can notice almost the same slope of the parts of the $Q_{\mathrm{sc}}\left(\Phi_{\mathrm{s}}\right)$ curves at the higher temperatures, both for $p$ - and $n$-type Si. Taking into account an approximation that in the case of the continuous distribution of the surface states $\mathrm{d} Q_{\mathrm{ss}} / \mathrm{d} \Phi_{\mathrm{s}}=-q^{2} N_{\mathrm{ss}}$, where $N_{\mathrm{ss}}$ is the surface state density (per unit energy) near the Fermi level, we have obtained for $N_{\mathrm{ss}}$ a value of an order of $10^{14} \mathrm{~m}^{-2} \mathrm{eV}^{-1}$.

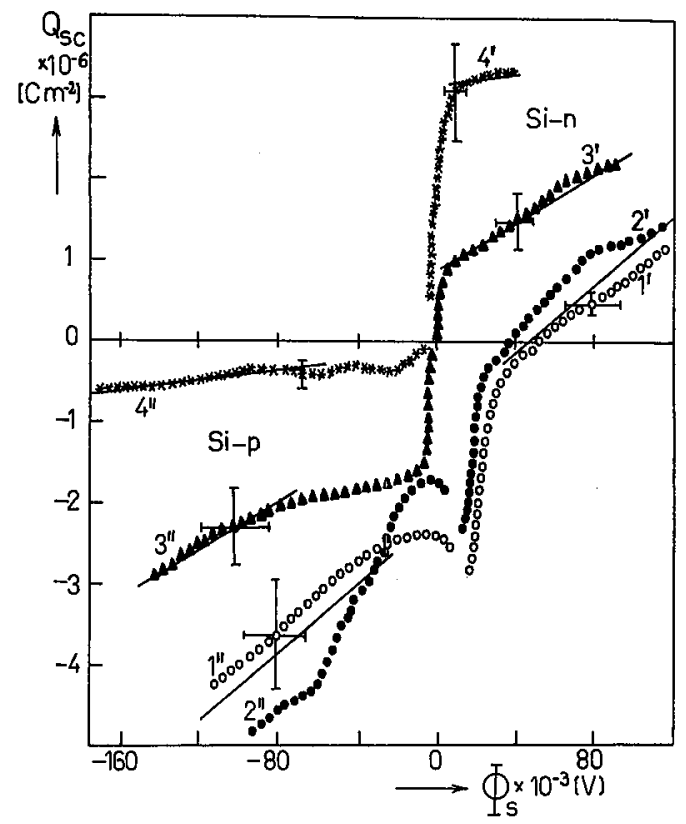

Fig. 4. Dependences of the surface space charge versus $\Phi_{\mathrm{s}}$ for $p$ - (") and $n$-type (') Si; etched surface (1), baked at $700 \mathrm{~K}(2), 920 \mathrm{~K}(3)$ and $970 \mathrm{~K}(4)$.

The abrupt change in $Q_{\mathrm{sc}}$ near the midgap points at the existence of another group of surface states, maybe of an impurity character [7].

The regular "moving" of the whole $Q_{\mathrm{sc}}\left(\Phi_{\mathrm{s}}\right)$ dependences toward more positive values after baking in vacuum proves that this process generates an additional positive charge connected with the thin oxide layer covering the surface.

All these effects are associated with the complex process of vacuum heating which causes not only dehydratation of the real $\mathrm{Si}$ surface but also oxidation and 
transformation of the native oxide covering the surface after chemical etching [8]. The more developed interpretation of this process will be done in the future.

\section{Conclusions}

The examination of the surface potential versus the surface Fermi level position, using the measurement of the surface photovoltage method, has yielded some information about the charge and the character of distribution of surface states on the real $\mathrm{Si}(111)$ surface.

Because all of the characteristics of $Q_{\mathrm{sc}}\left(\Phi_{\mathrm{s}}\right)$ are compatible for $p$ - and $n$-type material, we can come to the conclusion that the system of the electron states on these surfaces is mainly determined by the technological treatment, regardless of the type of bulk doping.

The work was sponsored by the Polish Ministry of National Education.

\section{References}

[1] C.E. Read, C.G. Scott; (Eds.), Surface Physics of Phosphors and Semicanductors, Açademic Press, London 1975.

[2] B. Adamowicz, S. Kochowski, in: Proc. 29th Inter. Wiss. Koll. TH llmenau, Ilmenau 1984, pp. 41-44.

[3] B. Adamowicz; Surf, Sci. 231, 1 (1990).

[4] N. Kasupke, M. Henzler, Surf. Sci. 54, 111 (1976).

[5] A. Ișmail, J.M. Palau, L. Lassabatere, Appl. Surf. Sci. 17, 363 (1984).

[6] A.Y. Rzhanov, Elektronnye Processy na Poverkhnosti Poluprovodnikov, Nauka, Moscow 1971 (in Russian).

[7] K. Heilig; H. Flietner, J. Reineke, J. Phyys. D, Appl. Phys, 12, 927 (1979).

[8] J. Szuber, Thin Solid Films 115, 27 (1984). 